\title{
Effect of sitting and lying Liuzijue exercise for pulmonary rehabilitation in acute exacerbation of chronic obstructive pulmonary disease patients with non-invasive ventilation: a randomized controlled trial
}

\author{
Shunqi Liao ${ }^{1}$, Fang Wang ${ }^{2}$, Qiao Lin $^{3}$, Feng Jian ${ }^{1}$, Yue Li ${ }^{1}$, Qiurun Zhong ${ }^{1}$, Yaling Huang ${ }^{1}$, Yang Lin ${ }^{1}$, \\ Han Wang ${ }^{1}$ \\ ${ }^{1}$ Chengdu University of Traditional Chinese Medicine, Chengdu, China; ${ }^{2}$ Hospital of Chengdu University of Traditional Chinese Medicine, \\ Chengdu, China; ${ }^{3}$ Department of Respiratory Medicine, Deyang People's Hospital, Deyang, China \\ Contributions: (I) Conception and design: S Liao, F Wang; (II) Administrative support: F Wang, Q Lin; (III) Provision of study materials or patients: \\ S Liao, F Jian, Y Li; (IV) Collection and assembly of data: All authors; (V) Data analysis and interpretation: S Liao, Q Zhong, Y Lin; (VI) Manuscript \\ writing: All authors; (VII) Final approval of manuscript: All authors. \\ Correspondence to: Fang Wang. Hospital of Chengdu University of Traditional Chinese Medicine, Chengdu, China. Email: wangf7640@163.com.
}

Background Acute exacerbation of chronic obstructive pulmonary disease (AECOPD) will lead to the
aggravation of respiratory symptoms and accelerate the progression of the disease. So early pulmonary
rehabilitation is essential for patients with AECOPD. We aimed to investigate the effect of sitting and lying
Liuzijue in AECOPD patients with non-invasive ventilation.

Methods: A total of 80 AECOPD patients with non-invasive ventilation were randomly divided into two groups. The controlled group (CG) $(n=40)$ received AECOPD conventional treatment and nursing, and the sitting and lying Liuzijue exercise group (LG) $(n=40)$ received sitting and lying Liuzijue therapy. Participants in the LG were required to take this training for 3 months, 30 minutes once every morning and evening. Pulmonary function test, 6-minute walking test (6MWT), Modified Medical Research Council Dyspnea Scales (mMRC), and the St George's Respiratory Questionnaire (SGRQ) were evaluated before and after the intervention. Blood gas was measured before treatment, 3 days, and 5 days after treatment. In addition, the length of hospital stay, the duration of non-invasive ventilation, and the frequency of acute exacerbation within 6 months after the intervention were compared.

Results: Seventy patients completed the study. The pulmonary function, 6MWT, mMRC scores, SGRQ, and $\mathrm{pH}$, were significantly improved in the LG. Meanwhile, the 6MWT of the CG was improved. The comparison between the groups showed that the improvement of the pulmonary function, $6 \mathrm{MWD}, \mathrm{mMRC}$ scores, SGRQ, the duration of non-invasive ventilation, and the frequency of acute exacerbation within 6 months of the LG group differed significantly from the CG.

Conclusions: Sitting and lying Liuzijue exercise methods significantly improved lung function, exercise endurance, and quality of life in AECOPD patients with non-invasive ventilation.

Trial Registration: Chinese Clinical Trial Registry ChiCTR2000034530.

Keywords: Sitting and lying Liuzijue; pulmonary rehabilitation; acute exacerbation of the chronic obstructive pulmonary disease (AECOPD)

Submitted Jul 22, 2021. Accepted for publication Sep 09, 2021.

doi: 10.21037/apm-21-2157

View this article at: https://dx.doi.org/10.21037/apm-21-2157 


\section{Introduction}

Chronic obstructive pulmonary disease (COPD) is a gradually worsening respiratory disease. COPD involves a very complex pathological process including destruction of lung architecture, alteration of the lung microenvironment and even promotion of lung cancer development (1). Studies have found that the tumor microenvironment in COPD can promote epithelial-mesenchymal transition (EMT) and suppress immune responses, leading to the development of lung cancer by promoting chronic inflammation and immune evasion (2). Patients with COPD rarely receive effective pulmonary rehabilitation exercises. This is related to the adverse systemic outcomes of COPD patients, including deterioration of cardiac and pulmonary function, low exercise tolerance, and susceptibility to infection (3).

COPD is often divided into stable stage and aggravating stage. Infection is recognized as a significant cause of acute exacerbation of chronic obstructive pulmonary disease (AECOPD) (4). According to epidemiological investigation, $22-40 \%$ of COPD patients have moderate or severe exacerbation at least once a year (3). AECOPD refers to the acute exacerbation of respiratory symptoms in patients with COPD that requires additional therapy $(5,6)$. Treatment of patients with AECOPD requires the use of bronchodilators, glucocorticoids, antibiotics, and other medications. Even breathing needs to be assisted with a ventilator (7). However, prolonged use of ventilators can aggravate respiratory muscle fatigue, which can lead to the occurrence of severe complications such as aggravating respiratory failure, and even pulmonary encephalopathy. Pulmonary rehabilitation is another avenue to help patients recover in addition to pharmacological treatment.

Pulmonary rehabilitation, mainly for exercise and health guidance, is recognized as an effective treatment for patients with COPD (8). It can promote the change of their behavior and long-term adherence. According to the European Respiratory Society/American Thoracic Society guidelines, early pulmonary rehabilitation exercise for AECOPD patients can combat skeletal muscle atrophy, improve exercise tolerance, improve lung function, reduce readmission rate, and improve quality of life (9). Traditional Chinese exercises (Tai Chi, Liuzijue, and Yi Jin Jing) effectively combine respiratory function training and body exercises. It is beneficial for the trainer's respiratory function, activity level, and physical and mental health (10). However, AECOPD patients mostly suffer from hypoxia with (or without) carbon dioxide retention and then combined with respiratory failure. Patients need to stay in bed for prolonged periods for non-invasive ventilation. The compliance of early getting out of bed is poor (11). Therefore, AECOPD patients with non-invasive ventilation need to find a simple, effective and convenient pulmonary rehabilitation method.

Liuzijue is a method of breathing adjustment and limb movement guided by the theory of traditional Chinese medicine. It can adjust the body's qi and blood, yin, and yang, promote qi and blood circulation, and restore the balance of yin and yang. According to the five Zang-organs to the five tones, the exerciser produces six sounds ( $\mathrm{xu}$, he, hu, si, chui, and xi) by expiration to adjust the corresponding internal organ functions (12). And Liuzijue has the characteristics of shrinking lip breathing and diaphragm breathing. It reduces the respiratory rate and delays the expiratory flow rate in COPD patients. Thus, it can prevent premature airway closure and improve abnormal breathing patterns $(13,14)$. Previous studies have shown that Liuzijue can effectively slow down the progressive decline of lung function in patients with COPD $(15,16)$ and enhance the flexibility of limbs' nerves and muscles as a traditional exercise focusing on regulating respiration (17). However, standing posture training will make it difficult for the old and weak or those who cannot stand for a long time. Therefore, Mr. Ma Li Tang specially compiled Liuzijue for sitting and lying. Sitting and lying Liuzijue is a lowintensity aerobic exercise based on standing Liuzijue, which is more suitable for AECOPD patients with long-term bedridden.

Earlier studies focused primarily on the effect of Liuzijue on patients with stable COPD, but were rarely used for AECOPD patients. Peijun Li's research (18) results show that Liuzijue combined with clinical guidance is beneficial to the pulmonary function, quality of life, and activity level of elderly COPD patients at home. In addition, Qigong (Wuqinxi, Liuzijue, Baduanjin, etc.) has a beneficial effect on relieving dyspnea, enhancing respiratory muscle strength, delaying the decline rate of lung function antiinfection ability (19). Most previous studies used Qigong in stable COPD patients with painless mobility. However, the latest guidelines recommend that early pulmonary rehabilitation is essential for patients with AECOPD. This research innovative application of the sitting and lying Liuzijue exercise in pulmonary rehabilitation treatment of AECOPD patients. Unlike previous ones, this study focused on AECOPD patients using ventilators, and the timing of the initiation of pulmonary rehabilitation was chosen after 
the pulmonary infection control window appeared.

The purpose of this study was to explore the effect of sitting and lying Liuzijue to provide a simple, implementable, and effective new method for early rehabilitation of pulmonary function in AECOPD patients with non-invasive ventilation. This study hypothesized that sitting and lying Liuzijue can significantly improve pulmonary function, activity tolerance, and quality of life in patients with AECOPD non-invasive ventilation. Concurrently, it was hypothesized that the duration of noninvasive ventilation and the frequency of acute exacerbation could be reduced. We present the following article in accordance with the CONSORT reporting checklist (available at https://dx.doi.org/10.21037/apm-21-2157).

\section{Methods}

\section{Research object}

AECOPD patients were recruited from the Hospital of Chengdu University of Traditional Chinese Medicine. Patients who met the inclusion criteria were randomly assigned to the two groups in a 1:1 allocation ratio. Because of the characteristics of the intervention, it was difficult to blind the exercise instructor or participants to their allocation. But the outcome assessor was blinded. The inclusion criteria were: they were between 40 and 80 years; COPD was diagnosed according to the Global Initiative for Chronic Obstructive Lung Disease (GOLD) guidelines, and was diagnosed as AECOPD (have acute worsening respiratory symptoms, including worsening dyspnea, worsening cough, increased sputum volume and/or purulent sputum) (8); AECOPD patients treated with non-invasive ventilation; Any regular exercise (at least 3 times a week) was not implemented within 6 months before this study; They could complete the sitting and lying Liuzijue exercise under guidance; All patients signed an informed consent form. Patients were excluded if they had severe cardiovascular, hepatorenal, and hematopoietic diseases, psychosis, or took part in other clinical trials. This single blind, randomized, controlled trial was approved by the Ethics Committee of the Hospital of Chengdu University of Traditional Chinese Medicine (No. 2019KL-064) and implemented following the Helsinki Declaration, which was amended in 2013.

\section{Intervention}

All subjects continued to receive their prescribed medications and non-invasive ventilation. According to the GOLD guidelines, the CG received routine nursing, including medication, smoking cessation, and life instructions (8). The LG chose the sitting and lying Liuzijue created by the martial arts master, Mr. Ma Litang, for exercise based on routine nursing (20) (Figure S1). After the lung infection control window appeared (21), the sitting and lying Liuzijue exercise began. During the 3-month intervention, LG exercised once in the morning and once in the evening for 30 minutes each time.

Before starting the sitting and lying Liuzijue exercise, the non-invasive ventilation mask was removed, and a nasal cannula was used for low flow oxygen inhalation (2$4 \mathrm{~L} / \mathrm{min}$ ). During exercise, the exercise intensity goal was to achieve $60-80 \%$ of the maximum heart rate. A pulse oximeter closely monitored the changes in heart rate and fingertip oxygen saturation. Patient's feeling was promptly questioned.

The program of LG included: (I) warm-up: patients adopted abdominal breathing to adjust breathing and gently stretched the limbs for 5 minutes; (II) sitting and lying Liuzijue exercise: patients performed complete sitting and lying Liuzijue, including six different expiratory tones ("Xu," "He," "Hu," "Si," "Cui," "Xi") and body activities for 20 minutes; (III) relaxation: patients adjusted breathing and relaxed the body for 5 minutes.

During the period of hospitalization, each participant sat and lied to do the Liuzijue exercise, and was completed under the guidance of respiratory doctors. Patients and their families were ensured to master the exercise methods before discharge fully. After leaving the hospital, patients were guided and supervised by daily telephone and weekly family visits. Patients were asked to document their exercise program, including exercise duration, location, intensity, etc.

\section{Outcome measures}

Blood gas analysis $\left(\mathrm{pH}, \mathrm{PaO}_{2}\right.$ and $\left.\mathrm{PaCO}_{2}\right)$ was performed before treatment, 3 days, and 5 days after the intervention. Lung function, $6 \mathrm{MWD}, \mathrm{mMRC}$, and quality of life were measured before the study and at 3 months. The length of hospital stay, the duration of non-invasive ventilation, and the frequency of acute exacerbations within 6 months after the intervention were also compared. Potential treatment effects were assessed by the frequency of acute exacerbations and quality of life. Acute exacerbation was defined as an unplanned visit to any hospital because of exacerbation 
of respiratory symptoms. After the intervention, monthly telephone calls evaluated the patient's status for 6 months. Lung function, $6 \mathrm{MWD}, \mathrm{mMRC}$, and quality of life were the primary outcome measures, and the others were secondary outcome measures.

\section{Pulmonary function tests}

Pulmonary function tests were performed following the American Thoracic Society/European Respiratory Society (ATS/ERS) guidelines. It was measured at the pulmonary function room of the Hospital of Chengdu University of Traditional Chinese Medicine by respiratory physicians. Each measurement was performed 3 times, and the optimal value was taken.

\section{6-minute walking test (6MWT)}

The $6 \mathrm{MWT}$ was tested in the corridor (30 meters long), specially designed by the respiratory department of our hospital. According to the guidelines of ATS, the respiratory doctors observed and recorded the patient's walking distance of the discomfort. The measurement accuracy was $0.5 \mathrm{~m}(22)$.

\section{Respiratory symptoms}

The symptoms of dyspnea were evaluated by the Modified Medical Research Council Dyspnea Scales (mMRC). The mMRC was initially proposed by experts in chronic bronchitis and emphysema. After years of application and improvement, mMRC is now commonly used to assess dyspnea in patients with COPD (23). It was divided into 04 levels; the higher the level, the more severe the breathing difficulties.

\section{Quality of life}

The quality of life was assessed by the St. George's Respiratory Questionnaire (SGRQ). The questionnaire was divided into 3 dimensions (symptoms, activity, and daily impact), which could well reflect the changes in exercise tolerance and quality of life in COPD patients (24). The total score of the questionnaire ranged from 0 to 100 points. The higher the score, the more severe the illness.

\section{Statistical analysis}

The outcome indicators for evaluating lung function in this study were based on $6 \mathrm{MWD}$, which is considered a simple and effective measure to reflect pulmonary rehabilitation. Based on the data used in previous studies (25), $54 \mathrm{~m}$ was considered the minimum significant difference between the group means, and the standard deviation (SD) of the measurement was $57 \mathrm{~m}$. PASS calculated the sample size 15.0 with $\alpha=0.05$ (two sides) and $\beta=0.02$ (power $80 \%$ ). The results showed a minimum sample size of 19 patients in each group. Based on our team's upfront findings, 30\% of patients could be lost, meaning there were at least 25 subjects in each group. 80 patients were finally enrolled to ensure that statistics were feasible.

All the data of this study were uniformly collated, summarized. Spss22.0 statistical software was used to analyze the data. Two-sided test criteria were used in this study. A P value $<0.05$ was statistically significant. Mean and SD described continuous variables. Categorical variables were described as absolute numbers and percentages. Within-group differences were compared using paired $t$-tests. Two independent samples $t$-test were used to analyze intergroup differences. Differences between the groups after the study were analyzed with the covariance model. For categorical data, the chi-square test analyzed the difference. Repeated measures ANOVA was used to examine daily changes of blood gas in the two groups.

\section{Results}

AECOPD patients were recruited from the Hospital of Chengdu University of Traditional Chinese Medicine from June 2018 to June 2020. A total of 80 AECOPD patients were enrolled. They were randomly divided into two groups: 40 in the CG and 40 in the LG. After the 9-month trial study, 70 (87.5\%) AECOPD patients completed the plan and were included in the analysis (Figure 1). The baseline characteristics of all two groups were shown in Table 1. There was no significant difference between the groups at baseline, and the two groups were well comparable. Similarly, there was no difference in general data, blood gas analysis, $6 \mathrm{MWD}, \mathrm{mMRC}$, and SGRQ scores between the two groups $(\mathrm{P}>0.05)$ (Table 1). In addition, based on the doctor's observations and participant feedback, no adverse reactions were found during the intervention period.

The repeated measurement analysis of variance (ANOVA) was used to analyze the $\mathrm{pH}, \mathrm{PaO}_{2}$, and $\mathrm{PaCO}_{2}$ of patients by 2 (group: CG and LG) $\times 3$ (test time: baseline before exercise, 3 days, and 5 days). The analysis results are shown in Table 2. The study found that only the effect of time on $\mathrm{pH}$ outcome was very significant $(\mathrm{P}<0.01$, $\mathrm{F}=13.254)$. The group's main effect of $\mathrm{pH}, \mathrm{PaO}_{2}$, and $\mathrm{PaCO}_{2}$ was not significant $(\mathrm{P}>0.05)$, and the interaction 


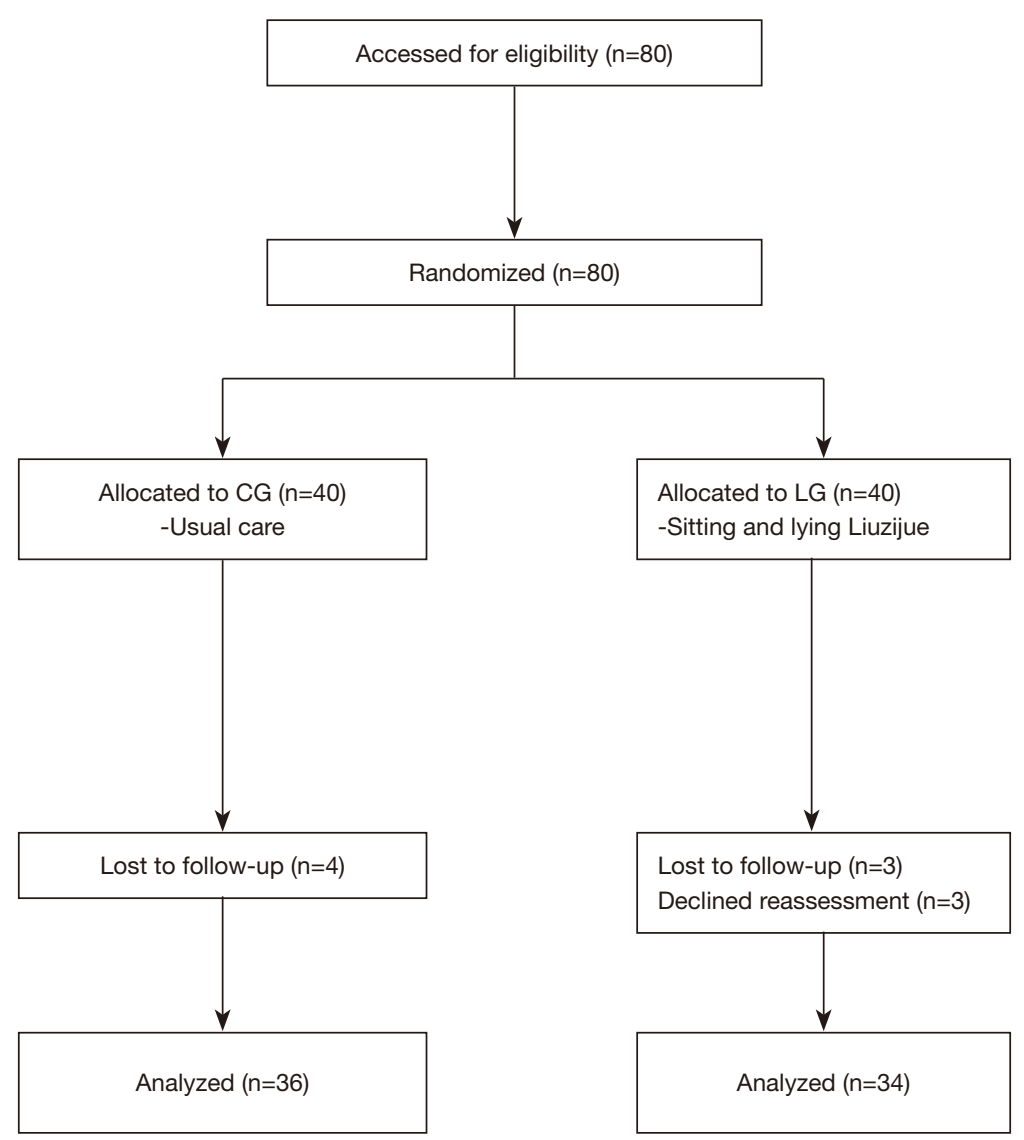

Figure 1 Study flow diagram. CG, control group; LG, sitting and lying Liuzijue exercise group.

between time and group was insignificant $(\mathrm{P}>0.05)$.

After 3 months of intervention, FEV1\% pred significantly improved in the LG patients $(\mathrm{P}=0.019)$, but $\mathrm{FVC} \%$ pred, FEV1/FVC\% pred did not improve significantly. There were no statistically significant differences in $\mathrm{FVC} \%$ pred, FEV1/FVC\% pred, and FEV1\%pred in the CG $(\mathrm{P}>0.05)$ (Table 3 and Figure 2). After the study, the dyspnea condition of the LG improved very significantly $(\mathrm{P}<0.01)$, whereas there was no change after study in patients of the CG. Assessment of exercise capacity, the 6MWD values of patients in the LG improved significantly after the intervention $(\mathrm{P}=0.002)$, and patients in the $\mathrm{CG}$ also improved after the intervention $(\mathrm{P}=0.047)$ (Table 3 and Figure 2). The comparison between the groups after the intervention showed that the improvement of the FEV1\% pred, 6MWD, mMRC scores of the LG differed significantly from the $\mathrm{CG}(\mathrm{P}=0.037, \mathrm{P}=0.013$, and $\mathrm{P}=0.012$, respectively) (Table 3 and Figure 2).

The scores of SGRQ in the LG were significantly decreased $(\mathrm{P}<0.001)$. The SGRQ score difference was not statistically significant in the CG. Comparing groups showed that the improvement in the total score and three domain scores of SGRQ was significantly different $(\mathrm{P}<0.001)$ (Table 4, Figure 3).

There was a significant difference in the duration of noninvasive ventilation between the CG and LG $(\mathrm{P}=0.041)$. The hospital stay of the two groups of patients was similar $(\mathrm{P}=0.763)$. The frequency of acute exacerbations within 6 months after the intervention was $0.41 \pm 0.07$ times in the CG and $0.23 \pm 0.09$ times in the LG, with significant differences between groups $(\mathrm{P}=0.035)$ (Table 5, Figure 4).

\section{Discussion}

The findings provide clinical evidence to recommend sitting and lying Liuzijue exercise for AECOPD patients with non-invasive ventilation, which can significantly improve pulmonary function, $6 \mathrm{MWD}, \mathrm{mMRC}$, and quality of life. Meanwhile, it can reduce the duration of non-invasive ventilation. After 6 months of follow-up, the patient's acute 
exacerbation frequency had decreased.

COPD causes not only airway and pulmonary diseases but also secondary systemic diseases. AECOPD will further accelerate the process of disease. GOLD (2020 Edition) (26) suggests that pulmonary rehabilitation with exercise as the main component should be recommended to patients with different levels of COPD because it is beneficial to COPD patients' physical health, activity level, respiratory function, and social communication. Its safety has been confirmed. However, there is no consistent conclusion as to when

Table 1 Baseline characteristics of participants

\begin{tabular}{|c|c|c|c|}
\hline Characteristics & $C G(n=36)$ & $L G(n=34)$ & $P$ value \\
\hline Sex, n (\%) & & & 0.452 \\
\hline Male & $27(75.0)$ & $28(82.4)$ & \\
\hline Female & $9(25.0)$ & $6(17.6 \%)$ & \\
\hline Age (years) & $61.21 \pm 7.38$ & $61.83 \pm 6.63$ & 0.665 \\
\hline BMI $\left(k g / m^{2}\right)$ & $21.15 \pm 3.74$ & $20.89 \pm 3.88$ & 0.741 \\
\hline Smoking history, n (\%) & & & 0.862 \\
\hline Yes & $28(77.8)$ & $27(79.4)$ & \\
\hline No & $8(22.2)$ & $7(20.6)$ & \\
\hline Smoking status, n (\%) & & & 0.692 \\
\hline Current smoker & $6(16.6)$ & $7(20.5)$ & \\
\hline Ex-smoker & $22(61.1)$ & $20(58.8)$ & \\
\hline Years of COPD & $9.75 \pm 3.44$ & $9.45 \pm 4.24$ & 0.701 \\
\hline \multicolumn{4}{|l|}{ Complications, n (\%) } \\
\hline Hypertension & $17(47.2)$ & $14(41.1)$ & 0.612 \\
\hline Diabetes & $6(16.6)$ & $6(17.6)$ & 0.913 \\
\hline $\begin{array}{l}\text { Coronary heart } \\
\text { disease }\end{array}$ & $7(19.4)$ & $4(11.7)$ & 0.326 \\
\hline
\end{tabular}

BMI, body mass index; CG, control group; LG, sitting and lying Liuzijue exercise group. exercise is best initiated after an acute exacerbation. The duration of symptoms of AECOPD was defined as 2 weeks after admission and 2-8 weeks as the recovery period. Yuan MX through literature analysis concluded that patients who implemented pulmonary rehabilitation in he recovery period all had varying degrees of prognostic improvement, while the prognosis in the symptomatic duration varied (27). This study started to implement pulmonary rehabilitation after the appearance of the pulmonary infection control window.

Liuzijue regulates Zang-Fu organs by regulating breathing, coordinating body movements, and regulating emotions. So, it plays a vital role in pulmonary diseases. A Previous study (15) found that the Liuzijue was used in AECOPD patients for pulmonary rehabilitation, improving respiratory muscle fatigue, respiratory symptoms, and exercise tolerance. Patients with AECOPD often cannot get out of bed early for pulmonary rehabilitation because of disease treatment, physical and psychological factors. Therefore, this study adopted the sitting and lying Liuzijue exercise, which is simple and easy to learn. The most important thing is that patients can complete the training in bed.

AECOPD with respiratory failure often needs to be treated with non-invasive ventilation, which can improve respiratory acidosis, reduce symptoms of dyspnea, etc. This study showed that $\mathrm{pH}$ improved significantly over time $(\mathrm{P}<0.01, \mathrm{~F}=13.254)$. Time had a positive effect on $\mathrm{PaCO}_{2}$ and $\mathrm{PaO}_{2}$, but the difference was insignificant. Our study followed the normal law of disease changes. Gold (2020 Edition) (26) pointed out that non-invasive ventilation can be stopped when the patient's condition improves and can tolerate at least 4 hours of non-assisted breathing. There were significant differences in the duration of noninvasive ventilation between the two groups after the intervention. This may be related to the fact that sitting and lying Liuzijue improves fatigue of respiratory muscles

Table 2 Comparisons of blood gas

\begin{tabular}{|c|c|c|c|c|c|c|c|c|c|}
\hline & \multicolumn{3}{|c|}{$C G(n=36)$} & \multicolumn{3}{|c|}{$L G(n=34)$} & $\begin{array}{l}\text { Time F } \\
\text { value }\end{array}$ & $\begin{array}{c}\text { Group F } \\
\text { value }\end{array}$ & $\begin{array}{c}\text { Time } \times \text { group } F \\
\text { value }\end{array}$ \\
\hline $\mathrm{pH}$ & $7.31 \pm 0.02$ & $7.32 \pm 0.02$ & $7.35 \pm 0.03$ & $7.31 \pm 0.01$ & $7.32 \pm 0.03$ & $7.35 \pm 0.02$ & $13.254^{\star \star}$ & 0.249 & 0.042 \\
\hline $\mathrm{PaO}_{2}$ & $58.69 \pm 8.15$ & $66.10 \pm 7.93$ & $74.70 \pm 5.08$ & $59.32 \pm 9.38$ & $67.60 \pm 6.24$ & $73.51 \pm 6.77$ & 1.515 & 0.202 & 0.010 \\
\hline $\mathrm{PaCO}_{2}$ & $64.73 \pm 7.82$ & $53.86 \pm 7.85$ & $49.73 \pm 8.27$ & $63.06 \pm 8.45$ & $52.90 \pm 7.96$ & $49.65 \pm 7.93$ & 1.636 & 0.052 & 0.020 \\
\hline
\end{tabular}

**, $\mathrm{P}<0.01$. CG, control group; LG, sitting and lying Liuzijue exercise group. 
Table 3 Comparisons of pulmonary function and exercise capacity

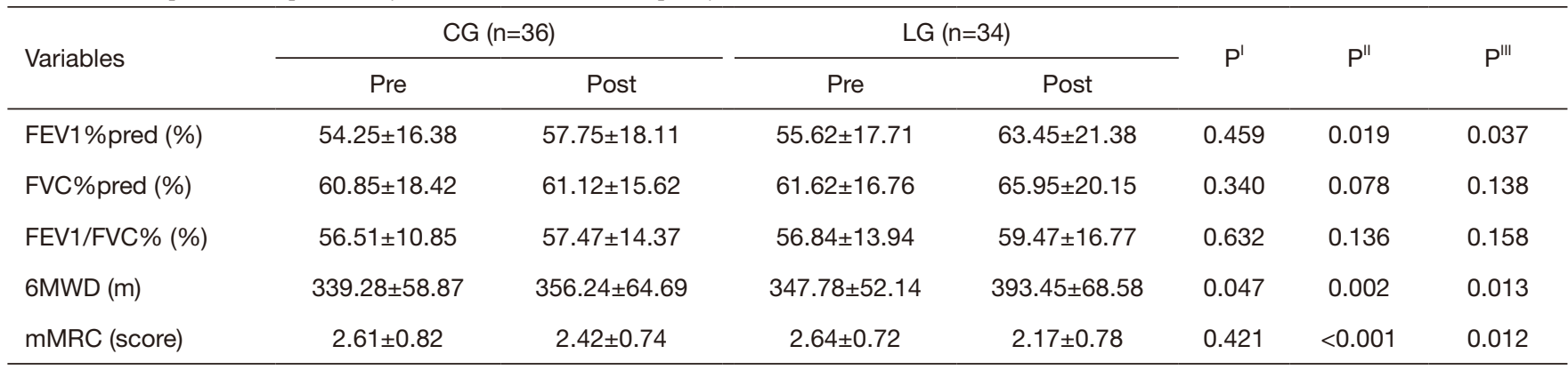

$\mathrm{P}^{\prime}$ values for comparison of outcome variables before to 3 months within the CG. $\mathrm{P}^{\prime \prime}$ values for comparison of outcome variables from before to 3 months within the LG. PIII values for the comparison of outcome variables from before to 3 months between groups. \%pred, percentage of predicted values; CG, control group; LG, sitting and lying Liuzijue exercise group; FEV1, forced expiratory volume in the first second; FVC, forced vital capacity; 6MWD, 6-min walking distance; mMRC, Modified Medical Research Council Dyspnea Scales.
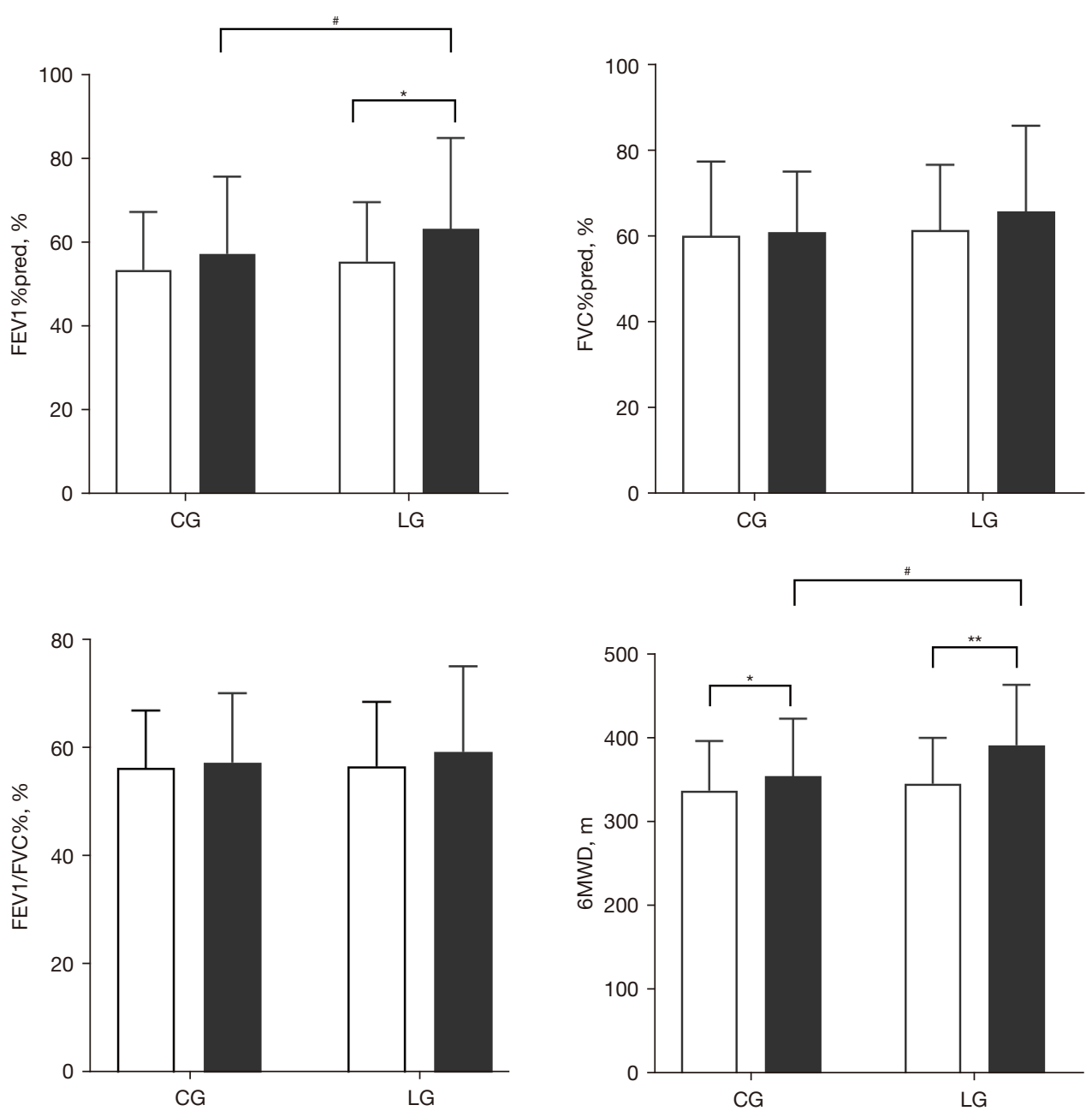

Figure 2 Pulmonary function and $6 \mathrm{MWD}$ in the CG and LG before and after the 3 -month intervention. ${ }^{*} \mathrm{P}<0.05$ within-group comparisons, and ${ }^{* *} \mathrm{P}<0.01$ within-group comparisons. ${ }^{*} \mathrm{P}<0.05$ intergroup comparisons. \% pred, percentage of predicted values; CG, control group; LG, sitting and lying Liuzijue exercise group; FEV1, forced expiratory volume in the first second; FVC, forced vital capacity; 6MWD, 6-min walking distance. 
Table 4 Scores of SGRQ

\begin{tabular}{|c|c|c|c|c|c|c|c|}
\hline Parameters & \multicolumn{2}{|c|}{$C G(n=36)$} & \multicolumn{2}{|c|}{$L G(n=34)$} & $P^{1}$ & $P^{\| \prime}$ & $P^{\prime \prime \prime}$ \\
\hline Symptom score & $60.88 \pm 16.72$ & $59.78 \pm 20.31$ & $58.68 \pm 18.45$ & $38.73 \pm 14.42$ & 0.745 & $<0.001$ & $<0.001$ \\
\hline Impact score & $31.36 \pm 12.28$ & $30.63 \pm 15.52$ & $32.16 \pm 15.12$ & $22.58 \pm 10.72$ & 0.591 & $<0.001$ & $<0.001$ \\
\hline Activity score & $51.35 \pm 22.35$ & $49.26 \pm 18.12$ & $50.75 \pm 19.64$ & $39.83 \pm 14.72$ & 0.274 & $<0.001$ & $<0.001$ \\
\hline
\end{tabular}

$P^{\prime}$ values for comparison of outcome variables before to 3 months within the CG. $P^{\prime \prime}$ values for comparison of outcome variables from before to 3 months within the LG. $P^{\text {III }}$ values for comparing outcome variables from before to 3 months between groups. CG, control group; LG, sitting and lying Liuzijue exercise group.
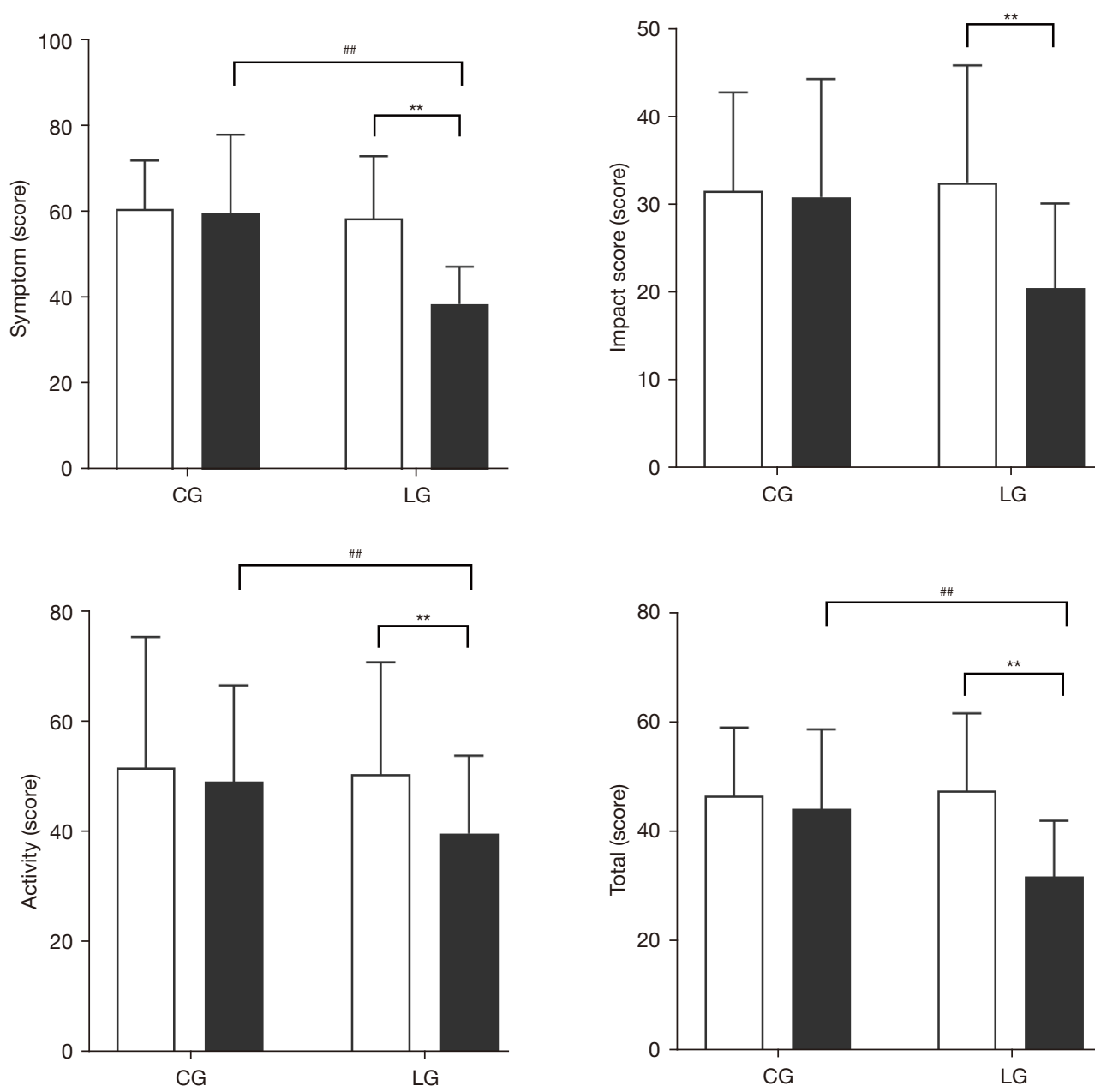

Figure 3 Domains of SGRQ in the CG and LG after the 3 -month intervention. ${ }^{* *} \mathrm{P}<0.01$ within-group comparisons, and ${ }^{\# \#} \mathrm{P}<0.01$ intergroup comparisons. CG, control group; LG, sitting and lying Liuzijue exercise group. 
Table 5 Comparisons of the duration of non-invasive ventilation, the hospital stay, and the frequency of acute exacerbations

\begin{tabular}{lccc}
\hline Variables & CG & LG & P value \\
\hline The duration of noninvasive ventilation (days) & $8.18 \pm 1.82$ & $7.28 \pm 2.10$ & 0.041 \\
The hospital stay (days) & $13.47 \pm 3.4$ & $12.96 \pm 4.7$ & 0.763 \\
The frequency of acute exacerbations (times) & $0.41 \pm 0.07$ & $0.23 \pm 0.09$ & 0.035 \\
\hline
\end{tabular}

CG, control group; LG, sitting and lying Liuzijue exercise group.
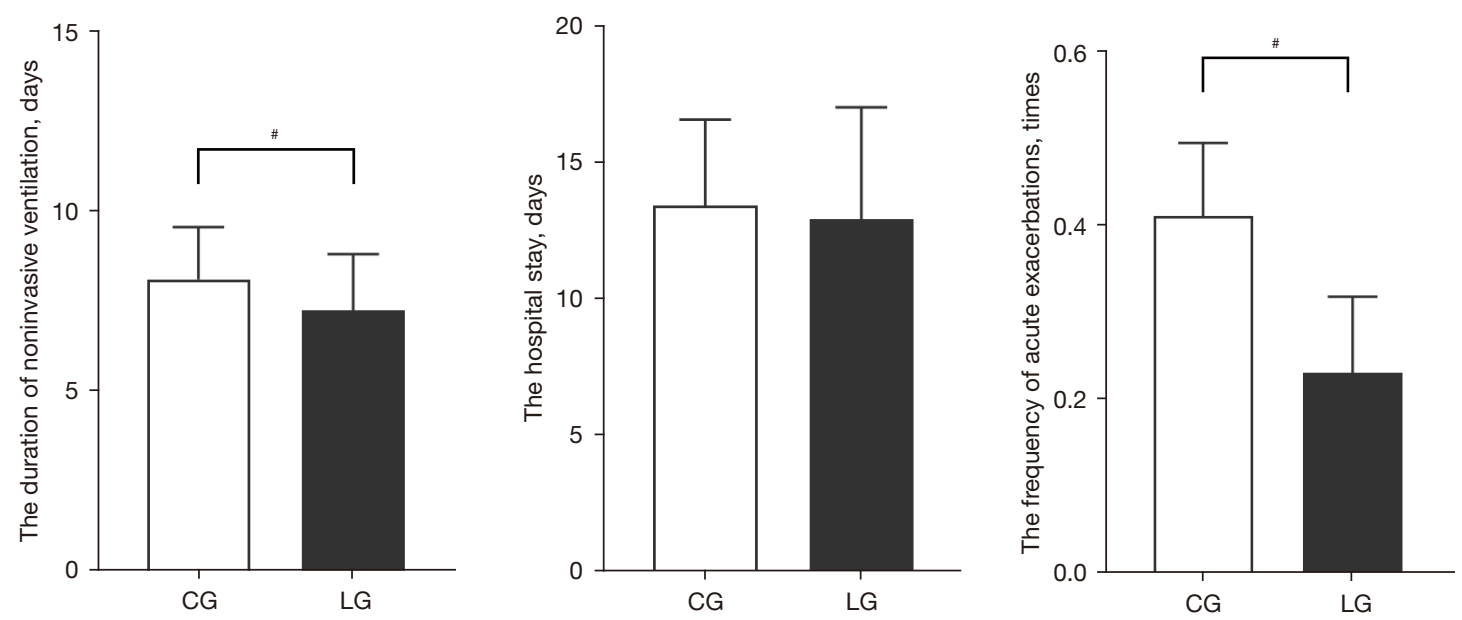

Figure 4 Comparisons of the duration of non-invasive ventilation, the hospital stay, and the frequency of acute exacerbations. ${ }^{\#} \mathrm{P}<0.05$ intergroup comparisons. CG, control group; LG, sitting and lying Liuzijue exercise group.

and reduces airway resistance in patients. Patients were followed-up for 6 months after the intervention. This study revealed that the patients in the LG had a significantly lower frequency of acute exacerbations than those in the CG. This may be related to the fact that sitting and lying Liuzijue has an improving effect on lung function and exercise tolerance. And after 3 months of sitting and lying Liuzijue exercise, the patient developed good habits related to regular exercise. However, this study did not account for different treatments after the intervention in the two groups of patients, limiting the result interpretation.

Lung function is considered the most crucial objective index to evaluate the deterioration of COPD. It declined rapidly in AECOPD (28-30). Previous studies showed $(31,32)$ that traditional Chinese medicine pulmonary rehabilitation can relieve the clinical symptoms of dyspnea in patients, enhance the level of respiratory muscle strength, and delay the rate of pulmonary function decline. Once again, a recent systematic review provided interesting evidence of Chinese traditional pulmonary rehabilitation in COPD treatment (33). The reason might be that traditional Chinese pulmonary rehabilitation training perfectly combines breathing and movement and focuses on mindfulness movement.

Interestingly, a previous study (34) showed that mindfulness movement could effectively improve the patients' willingness to exercise and relieve the negative experience of patients. The above results could also be found in this study that FEV1\% pred was significantly increased in LG patients. The results showed that sitting and lying Liuzijue can improve lung function during non-invasive ventilation in AECOPD patients. This was consistent with the results of previous studies using Liuzijue interventions in patients with the stable stage of COPD (18). Compared with the abdominal-retracting lip breathing method, the sitting and lying Liuzijue adopts the inverse abdominal breathing method combined with physical activities, which can effectively exercise the diaphragm and chest auxiliary respiratory muscles. Therefore, the patient's breathing time is prolonged, and the breathing depth increases. To achieve the purpose of increasing the gas exchange rate of the lungs and increasing the lung capacity. 
Dyspnea is the hallmark clinical symptom of COPD patients. Because of dyspnea, COPD patients often suffer from physical discomfort from exercise. Therefore, they are often reluctant to exercise. This is because extra breathing muscles are required when exercising. This can easily lead to fatigue and weakness of respiratory muscles. This causes the patient's dyspnea to worsen. Patients with COPD stay in a state of dyspnea for a long time, which leads to anxiety and emotional tension. A bad mental state further aggravates the patient's dyspnea. A Previous study (17) found Liuzijue improved symptoms of dyspnea in COPD patients. Like previous studies, this study showed that the mMRC scores of LG patients decreased significantly after 3 months of intervention, and the improvement of dyspnea in LG was better than CG.

Pulmonary rehabilitation consists primarily of exercise training and respiratory training. A Cochrane evidencebased analysis confirmed the effect of pulmonary rehabilitation on improving health-related aspects in AECOPD patients (35). The results of a previous study (36) showed that the $6 \mathrm{MWD}$ of COPD patients increased 49 meters from baseline after pulmonary rehabilitation. Another study found (37) high-intensity aerobic exercise can improve the respiratory function of COPD patients and reduce the risk of cardiovascular disease. Chinese traditional pulmonary rehabilitation consists primarily of moderate-intensity and low-intensity aerobic training, which can effectively improve 6MWD in COPD patients. Following previous studies, the results of this study showed a significant difference in $6 \mathrm{MWD}$ between two groups after intervention. The 6MWD increased significantly after sitting and lying Liuzijue exercise. It shows that sitting and lying Liuzijue can significantly improve the exercise capacity of ANCOPD patients with non-invasive ventilation. This may be because the exercises of the sitting and lying Liuzijue require breathing exercises and exercises of limbs. Exercises of the limbs include rhythmic flexion and extension of the upper and lower extremities, both to exercise limb flexibility and enhance muscle endurance.

Quality of life relates to curing many diseases, and improving patients' quality of life has become a nonnegligible problem during treatment. A previous study (38) have shown that the total SGRQ score, symptoms, and activity scores of patients with stable COPD after 3 months of Liuzijue exercise were significantly lower than those of the control group, and there was no significant difference in the daily impact scores. In this study, the total score of SGRQ, symptoms, activity, and daily impact scores in the LG were significantly lower than those in the CG. This result is better than the results of previous studies on Liuzijue for COPD patients. This may be because of the different severity of the disease. Patients with AECOPD need to be hospitalized and rested in bed, and the patients' symptoms are more severe, which increases the patient's anxiety and concerns about the prognosis of the disease. The sitting and lying Liuzijue exercise allows the patient to focus and be calm. The patient received psychological adjustment and physical exercise simultaneously. This has a more significant impact on the daily life of AECOPD patients. Physical activity also plays a vital role in daily life. Studies have found that patients' upper limb muscle strength with COPD was related to exercise capacity and affected quality of life (39). Each breathing exercise of sitting and lying Liuzijue is equipped with corresponding upper limb exercises.

The main strength of this study is that an early pulmonary rehabilitation program in patients with AECOPD can be achieved. Sitting and lying Liuzijue is a low-intensity aerobic exercise that can be done in bed without special equipment. And it skillfully combines breathing exercise and skeletal muscle exercise. This makes sitting and lying Liuzijue feasible for non-invasive ventilation patients with AECOPD. After the intervention, the 6-month follow-up can observe the long-term effect. This study also has some limitations. First, patients come from the same hospital. Second, the sample size is relatively small. Third, the blind method cannot be achieved. Therefore, in order to further analyze the effect of sitting and lying Liuzijue exercise, it is necessary to further accumulate clinical cases and improve the overall understanding of the mechanism of this effect. Fourth, Sitting and lying Liuzijue is not lively and interesting enough, which can reduce the patient's compliance. Finally, because the sitting and lying Liuzijue movement is a meditative movement, its effect may be limited by the comprehension and acceptance of people from different cultural backgrounds. Our future trials will focus on long-term follow-up investigations of patients. The potential therapeutic effects of the sitting and lying Liuzijue exercise were evaluated by the frequency of acute exacerbations, quality of life, exercise habits, skeletal muscle and respiratory muscle function.

\section{Conclusions}

Sitting and lying Liuzijue can improve pulmonary function, dyspnea, exercise endurance, and quality of life in AECOPD 
patients with non-invasive ventilation. Meanwhile, it can reduce the duration of non-invasive ventilation and the frequency of acute exacerbations. Therefore, sitting to lying Liuzijue exercise is a recommended rehabilitation modality for AECOPD patients with non-invasive ventilation. Further studies should focus on the mechanism of sitting to lying Liuzijue exercise in AECOPD patients with noninvasive ventilation.

\section{Acknowledgments}

Funding: This study was supported by a grant from the Science and Technology Department of Sichuan Province (2019YFS0391).

\section{Footnote}

Reporting Checklist: The authors have completed the CONSORT reporting checklist. Available at https://dx.doi. org/10.21037/apm-21-2157

Trial Protocol: Available at https://dx.doi.org/10.21037/apm21-2157

Data Sharing Statement: Available at https://dx.doi. org/10.21037/apm-21-2157

Conflicts of Interest: All authors have completed the ICMJE uniform disclosure form (available at https://dx.doi. org/10.21037/apm-21-2157). The authors have no conflicts of interest to declare.

Ethical Statement: The authors are accountable for all aspects of the work in ensuring that questions related to the accuracy or integrity of any part of the work are appropriately investigated and resolved. The study was conducted in accordance with the Declaration of Helsinki (as revised in 2013). The Ethics Committee approved the study protocols of the Hospital of Chengdu University of Traditional Chinese Medicine (2019KL-064). All participants obtained informed written consent.

Open Access Statement: This is an Open Access article distributed in accordance with the Creative Commons Attribution-NonCommercial-NoDerivs 4.0 International License (CC BY-NC-ND 4.0), which permits the noncommercial replication and distribution of the article with the strict proviso that no changes or edits are made and the original work is properly cited (including links to both the formal publication through the relevant DOI and the license). See: https://creativecommons.org/licenses/by-nc-nd/4.0/.

\section{References}

1. Vozoris NT. Opioid utility for dyspnea in chronic obstructive pulmonary disease: a complicated and controversial story. Ann Palliat Med 2020;9:571-8.

2. Hou W, Hu S, Li C, et al. Cigarette Smoke Induced Lung Barrier Dysfunction, EMT, and Tissue Remodeling: A Possible Link between COPD and Lung Cancer. Biomed Res Int 2019;2019:2025636.

3. Singh D, Agusti A, Anzueto A, et al. Global Strategy for the Diagnosis, Management, and Prevention of Chronic Obstructive Lung Disease: the GOLD science committee report 2019. Eur Respir J 2019;53:1900164.

4. Liu JX, Li M, Li HZ, et al. Early goal-directed sedation in the treatment of AECOPD patients with NIPPV:a pilot feasibility trial. China J Mod Med 2020;30:98-103.

5. Koo HK, Vasilescu DM, Booth S, et al. Small airways disease in mild and moderate chronic obstructive pulmonary disease: a cross-sectional study. Lancet Respir Med 2018;6:591-602.

6. Jiang W, Chao Y, Wang X, et al. Day-to-Day Variability of Parameters Recorded by Home Noninvasive Positive Pressure Ventilation for Detection of Severe Acute Exacerbations in COPD. Int J Chron Obstruct Pulmon Dis 2021;16:727-37.

7. Mathioudakis AG, Janssens W, Sivapalan P, et al. Acute exacerbations of chronic obstructive pulmonary disease: in search of diagnostic biomarkers and treatable traits. Thorax 2020;75:520-7.

8. GOLD.org [Internet]. Global Initiative for Chronic Obstructive Lung Disease (GOLD): Global strategy for the diagnosis, management, and prevention of chronic obstructive pulmonary disease; c2017 [cited 2021 April 25]. Available online: http://www.goldcopd.org

9. Wedzicha JA Ers Co-Chair, Miravitlles M, Hurst JR, et al. Management of COPD exacerbations: a European Respiratory Society/American Thoracic Society guideline. Eur Respir J 2017;49:1600791.

10. Wu W, Liu X, Liu J, et al. Effectiveness of waterbased Liuzijue exercise on respiratory muscle strength and peripheral skeletal muscle function in patients with COPD. Int J Chron Obstruct Pulmon Dis 2018;13:1713-26.

11. Zhang L, Yan R, Zhou Y, et al. Application of early 
exercise safety management in patients undergoing mechanical ventilation in intensive care unit. Zhonghua Wei Zhong Bing Ji Jiu Yi Xue 2020;32:840-5.

12. Leung RW, McKeough ZJ, Peters MJ, et al. Short-form Sun-style t'ai chi as an exercise training modality in people with COPD. Eur Respir J 2013;41:1051-7.

13. de Araujo CL, Karloh M, Dos Reis CM, et al. Pursedlips breathing reduces dynamic hyperinflation induced by activities of daily living test in patients with chronic obstructive pulmonary disease: A randomized cross-over study. J Rehabil Med 2015;47:957-62.

14. Xiao CM, Zhuang YC. Efficacy of Liuzijue Qigong in Individuals with Chronic Obstructive Pulmonary Disease in Remission. J Am Geriatr Soc 2015;63:1420-5.

15. Wang ZW, Yang PL, Tang J. The Randomized Controlled Trial of TCM Pulmonary Rehabilitation Exercise on COPD Patients Pulmonary Function and Acute Exacerbation Rate. J Emerg Tradit Chin Med 2013;22:23-4, 58.

16. Li H, Lu ZQ, Liang RY. Research progress of chronic obstructive pulmonary disease combined with cardiovascular disease. J Clin Pulm Med 2017;04:154-9.

17. Wang ZW, Chen LJ, Yang P, et al. Randomized Controlled Study of Early Intervention with TCM Pulmonary Rehabilitation in AECOPD Patients. J Emerg Tradit Chin Med 2011;20:1734-6.

18. Li P, Liu J, Lu Y, et al. Effects of long-term homebased Liuzijue exercise combined with clinical guidance in elderly patients with chronic obstructive pulmonary disease. Clin Interv Aging 2018;13:1391-9.

19. Liang LP. Effects of multiple traditional health-care sports programs on psychoemotional and immune functions of middle-aged and elderly people. Chin J Gerontol 2018;38:418-20.

20. Ma LT. The authentic Health Qigong of Ma Li Tang. Beijing: Beijing People's Sports Publishing House, 1993.

21. Qi LM. Clinical effect of sequential non- invasive and invasive mechanical ventilation on severe pneumonia guided by pulmonary-infection-control-window. Pract J Card Cereb Pneum Vasc Dis 2016;24:103-5.

22. ATS Committee on Proficiency Standards for Clinical Pulmonary Function Laboratories. ATS statement: guidelines for the six-minute walk test. Am J Respir Crit Care Med 2002;166:111-7.

23. Han MK, Muellerova H, Curran-Everett D, et al. GOLD 2011 disease severity classification in COPDGene: a prospective cohort study. Lancet Respir Med 2013;1:43-50.
24. Jones PW. St. George's Respiratory Questionnaire: MCID. COPD 2005;2:75-9.

25. Ng BH, Tsang HW, Jones AY, et al. Functional and psychosocial effects of health qigong in patients with COPD: a randomized controlled trial. J Altern Complement Med 2011;17:243-51.

26. GOLD.org [Internet]. Global Initiative for Chronic Obstructive Lung Disease (GOLD): Global strategy for the diagnosis, management, and prevention of chronic obstructive pulmonary disease (2020 report); c2020 [cited 2021 April 25]. Available online: https://goldcopd.org/ gold-reports/

27. Yuan MX, Chen YS, Ou XM, et al. Research progress of pulmonary rehabilitation in acute exacerbation of chronic obstructive pulmonary disease. Chin J Respir Crit Care Med 2020;19:92-5.

28. Chan AW, Lee A, Suen LK, et al. Tai chi Qigong improves lung functions and activity tolerance in COPD clients: a single blind, randomized controlled trial. Complement Ther Med 2011;19:3-11.

29. Stav D, Raz M, Shpirer I. Three years of pulmonary rehabilitation: inhibit the decline in airflow obstruction, improves exercise endurance time, and body-mass index, in chronic obstructive pulmonary disease. BMC Pulm Med 2009;9:26.

30. Güell MR, Cejudo P, Ortega F, et al. Benefits of LongTerm Pulmonary Rehabilitation Maintenance Program in Patients with Severe Chronic Obstructive Pulmonary Disease. Three-Year Follow-up. Am J Respir Crit Care Med 2017;195:622-9.

31. Zang M, Cai GL, Lin WB, et al. Influences of Wuqinxi Bird Exercises Combined with Simple Breath Exercises on Quality of Life and Immune Function of Patients with Chronic Obstructive Pulmonary Disease. J Guangzhou Univ Tradit Chin Med 2017;034:819-23.

32. Liu X, Li P, Xiao L, et al. Effects of home-based prescribed pulmonary exercise by patients with chronic obstructive pulmonary disease: study protocol for a randomized controlled trial. Trials 2019;20:41.

33. Luo X, Zhang J, Castelberg R, et al. The Effects of Traditional Chinese Exercise in Patients with Chronic Obstructive Pulmonary Disease: A Meta-Analysis. PLoS One 2016;11:e0161564.

34. Wielgosz J, Goldberg SB, Kral TRA, et al. Mindfulness Meditation and Psychopathology. Annu Rev Clin Psychol 2019;15:285-316.

35. Puhan MA, Gimeno-Santos E, Cates CJ, et al. Pulmonary rehabilitation following exacerbations of chronic 
obstructive pulmonary disease. Cochrane Database Syst Rev 2016;12:CD005305.

36. He M, Yu S, Wang L, et al. Efficiency and safety of pulmonary rehabilitation in acute exacerbation of chronic obstructive pulmonary disease. Med Sci Monit 2015;21:806-12.

37. Mohammed J, Derom E, Van Oosterwijck J, et al. Evidence for aerobic exercise training on the autonomic function in patients with chronic obstructive pulmonary disease (COPD): a systematic review. Physiotherapy 2018;104:36-45.

38. Wang ZW, Yang PL, Tang J, et al. Effects of "Six Letters

Cite this article as: Liao S, Wang F, Lin Q, Jian F, Li Y, Zhong Q, Huang Y, Lin Y, Wang H. Effect of sitting and lying Liuzijue exercise for pulmonary rehabilitation in acute exacerbation of chronic obstructive pulmonary disease patients with non-invasive ventilation: a randomized controlled trial. Ann Palliat Med 2021;10(9):9914-9926. doi: 10.21037/apm-212157
Formula" on quality of life and syndrome of traditional Chinese medicine in patients with chronic obstructive pulmonary disease. Shanghai J Tradit Chin Med 2010;44:54-6, 9.

39. Kaymaz D, Candemir İÇ, Ergün P, et al. Relation between upper-limb muscle strength with exercise capacity, quality of life and dyspnea in patients with severe chronic obstructive pulmonary disease. Clin Respir J 2018;12:1257-63.

(English Language Editor: J. Chapnick) 

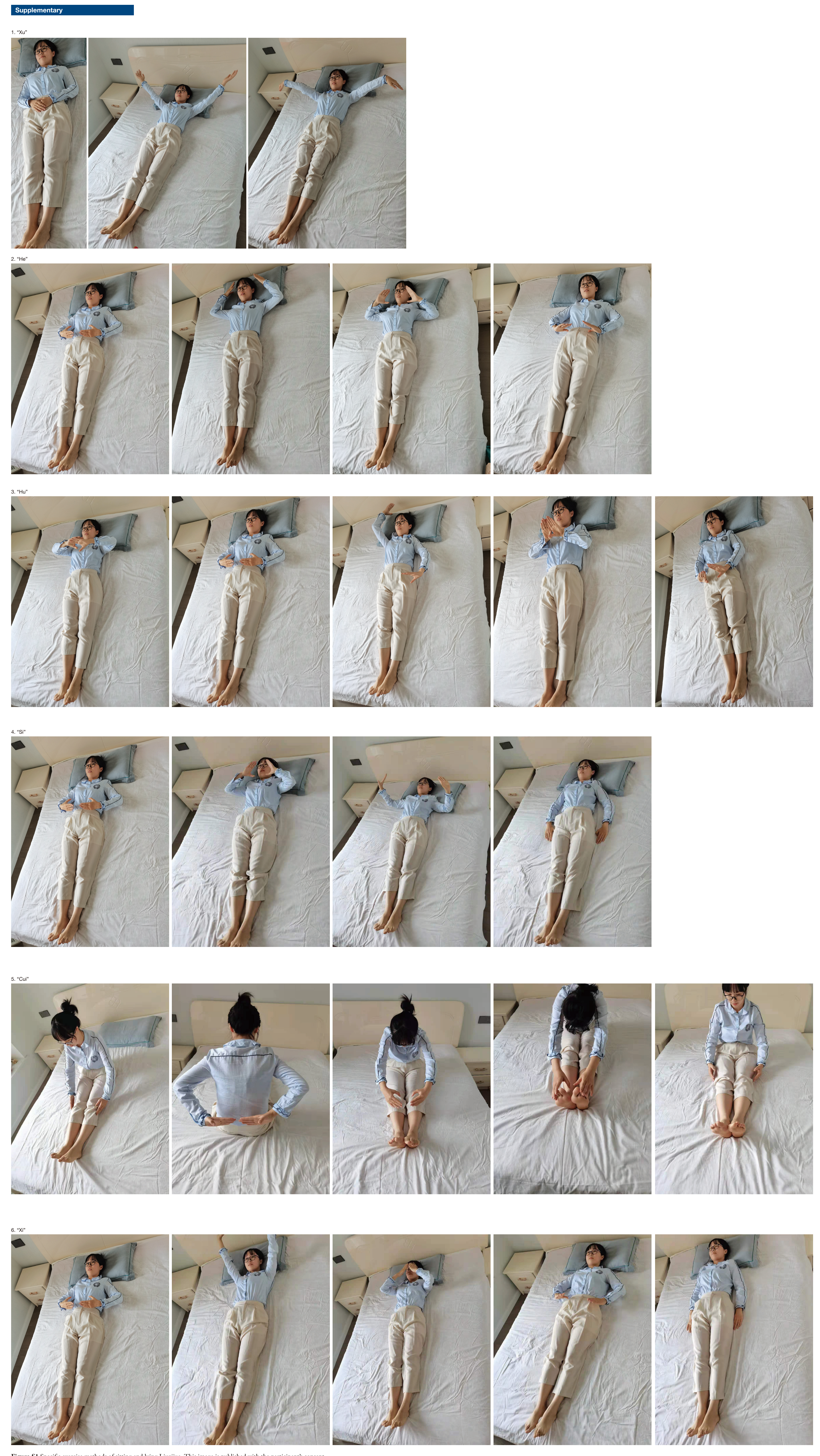